\title{
Electrophoretic Deposition of Chitosan/Eudragit E 100/AgNPs Coatings for Controlled Release of Antibacterial Substance
}

\author{
Lukasz Pawłowski ${ }^{1}$, Andrzej Zieliński ${ }^{1}$ \\ ${ }^{1}$ Institute of Machine and Material Technology/Gdańsk University of Technology \\ Narutowicza 11/12, 80-233 Gdańsk, Poland \\ lukasz.pawlowski@pg.edu.pl; andrzej.zielinski@pg.edu.pl
}

\section{Extended Abstract}

The development of bacterial infection of the tissues surrounding an implant is one of the leading causes of implant surgery failure. In order to prevent the deposition of bacteria on the implant surface and the formation of biofilm, coatings that exhibit antibacterial properties are manufactured. However, the problem is the controlled release of the therapeutic substance from the coating over the extended life of the implant [1], [2]. Usually, the active substance is released immediately when the implant is inserted into an environment simulating human body fluids, which results in burst release phenomenon, namely, the release of a large dose of active substance in a short period of time, which may result in a toxic effect.

Unfortunately, chitosan, one of the most commonly used biopolymers in controlled drug delivery systems, is unstable in neutral $\mathrm{pH}$ environments, readily absorbs water, and swells, resulting in rapid release of the therapeutic agent. Therefore, it is necessary to limit the dissolution rate of the chitosan coating at neutral $\mathrm{pH}$. Previous studies [3] have confirmed that the addition of Eudragit E 100, a cationic copolymer based on dimethylaminoethyl methacrylate, butyl methacrylate, and methyl methacrylate in a 2:1:1 ratio, to chitosan coatings significantly reduced the degradation of these coatings in artificial saliva solution at neutral $\mathrm{pH}$ while maintaining high sensitivity to $\mathrm{pH}$ drop. Hence, a biopolymer matrix of this type should provide a good carrier for an active substance, such as metallic nanoparticles with antibacterial activity, which will be released only when inflammation of peri-implant tissues occurs.

The scientific objective of this study was to fabricate a smart composite coating of chitosan/Eudragit E 100/silver nanoparticles (chit/EE100/AgNPs) on grade 2 titanium surface using variable deposition parameters such as AgNPs concentration, voltage, and deposition time by the electrophoretic method. The determinant of the process of creating such composite coatings with EE100 by electrophoretic method on the surface of Ti grade 2 has not been determined yet. No publication concerning investigations of coatings containing chitosan/EE100 with silver nanoparticles has been found. The microstructure, surface roughness, thickness, chemical and phase composition, mechanical and electrochemical properties, silver release rate at different $\mathrm{pH}$, and wettability of the produced coatings were investigated.

The chit/EE100/AgNPs composite coatings were successfully deposited on titanium substrates by one-step electrophoretic deposition. Using lower values of deposition parameters, coatings with more uniform morphology were obtained. The proposed system based on chitosan and Eudragit E 100 with the addition of silver nanoparticles exhibited good corrosion resistance, adequate wettability, and showed high sensitivity to reduced $\mathrm{pH}$ environment, which is important for applications in controlled drug delivery systems. However, the adhesion of these coatings to the metallic substrate was insufficient. The addition of Eudragit E 100 did not significantly improve the mechanical properties of the chitosan coatings. The tendency of silver nanoparticles to form agglomerates was also problematic, despite the addition of the dispersing agent.

\section{References}

[1] Ł. Pawłowski, "pH-dependent composite coatings for controlled drug delivery system - Review," Inżynieria Mater., vol. 1, no. 3, pp. 4-9, 2019, doi: 10.15199/28.2019.3.1.

[2] M. Bartmański, Ł. Pawłowski, A. Zieliński, A. Mielewczyk-Gryń, G. Strugała, and B. Cieslik, "Electrophoretic deposition and characteristics of chitosan / nanosilver composite coatings on the nanotubular TiO 2layer," Coatings, pp. 1-18, 2020, doi: 10.3390/coatings10030245.

[3] Ł. Pawłowski, M. Bartmański, G. Strugała, A. Mielewczyk-Gryń, M. Jażdżewska, and A. Zieliński, "Electrophoretic Deposition and Characterization of Chitosan/Eudragit E 100 Coatings on Titanium Substrate," Coatings, vol. 10, no. 7, p. 607, Jun. 2020, doi: 10.3390/coatings 10070607. 